\title{
CÁC YẾU TỐ ẢNH HƯỞNG ĐẾN Ý ĐỊNH SỬ DỤNG HÓA ĐƠN ĐIỆN TỬ CỦA DOANH NGHIỆP TẠI CHI CỤC THUẾ QUẬN TÂN PHÚ - TP.HCM
}

\author{
NGUYỄN MINH TUÂN ${ }^{1}$, ĐỖ VIỄN CHÂU ${ }^{2}$ \\ ${ }^{1}$ Khoa Quản trị kinh doanh, Trường Đại học Công nghiệp thành phố Hồ Chí Minh \\ ${ }^{2}$ Chi Cục Thuế Quận Tân Phú TP.HCM \\ nguyenminhtuan@iuh.edu.vn
}

Tóm tắt. Nội dung chính của nghiên cứu là tìm hiểu và xác định các yếu tố chính ảnh hưởng đến ý định sử dụng hóa đơn điện tử của doanh nghiệp tại Chi Cục Thuế quận Tân Phú TP.HCM. Trong nghiên cứu, tác giả đề xuất mô hình nghiên cứu gồm 6 yếu tố là tính hữu ích $(\mathrm{HI})$, dễ sử dụng $(\mathrm{SD})$, tính tương hổ $(\mathrm{TH})$, chi phí $(\mathrm{CP})$, sự tin tưởng $(\mathrm{TT})$, chuẩn chủ quan $(\mathrm{CQ})$. Kết quả nghiên cứu có 6 yếu tố ảnh hưởng đến ý định sử dụng hóa đơn điện tử của doanh nghiệp tại Chi Cục Thuế quận Tân Phú TP.HCM theo mức độ ảnh hưởng từ cao đến thấp là tính hữu ích, dễ sử dụng, chuẩn chủ quan, sự tin tưởng, tính tương hổ

Từ khoá: Hóa đơn điện tử, chuẩn chủ quan, hành vi khách hàng, ý định

\section{ENTERPRISE INTENT ON USING ELECTRONIC INVOICES AT TAN PHU DISTRICT TAX DEPARTMENT IN HO CHI MINH CITY}

\begin{abstract}
The main content of the study is to find out and identify the main factors affecting enterprise intent on using electronic invoices at Tan Phu District Tax Department in Ho Chi Minh City. In the study, the author proposes a research model consisting of 6 factors: (1) Usefulness, (2) ease of use, (3) trust, (4) price, (5) reciprocity, (6) subjective standards. The research results have 5 main factors affecting enterprise intent on using electronic invoices at Tan Phu District Tax Department in Ho Chi Minh City anh they are arranged in order of impact from high to low, such as Perceived utility, Perceived use, Subject standard, Perceived trusth, and Perceived reciprocity
\end{abstract}

Key words: customer behavior, consumer intent, electronic invoices, behavior perceive

\section{1. ĐẠT VẤN Đî̀}

Trong bối cảnh cuộc cách mạng công nghiệp 4.0 đã thực sự bắt đầu, thể hiện rõ nét nhất sự phát triển mạnh mẽ của công nghệ số, kinh tế số, công nghệ thông tin và internet, sự tích hợp của nhiều công nghệ hiện đại trong nhiều lĩnh vực vật lý, hóa học, sinh học với công nghệ thông tin giữ vai trò chi phối và là trung tâm kết nối, Hóa đơn điện tử (HĐĐT) đã ra đời và dần dần phát triển mạnh mẽ ở nhiều quốc gia thay thế hóa đơn giấy truyền thống.

Tuy vậy, với sự phát triển tự nhiên vốn có của nó, HĐĐT chưa được sử dụng rộng rãi ở Việt Nam bất chấp hình thức hóa đơn này có rất nhiều ưu điểm so với hóa đơn giấy truyền thống. Điều này đặt ra một đòi hỏi cấp thiết, cần nghiên cứu giải pháp để thúc đẩy sử dụng HĐĐT ở Việt Nam. Cùng với sự phát triển HĐĐT thì cần hình thành cơ sở dữ liệu quốc gia về HĐĐT và các giao dịch kinh doanh của người nộp thuế nhằm hỗ trợ cho hoạt động cung cấp, sử dụng, kiểm tra đối chiếu thông tin HĐĐT của người nộp thuế và hoạt động quản lý nhà nước của cơ quan thuế và các cơ quan chức năng khác.

Quận Tân Phú đang rất phát triển, số lượng doanh nghiệp trên 16.000 doanh nghiệp, thành lập mới không ngừng tăng. Do đó, công tác quản lý về hóa đơn tại Chi cục Thuế quận Tân Phú rất được quan tâm. Vì vậy, việc nghiên cứu tìm hiểu ý định sử dụng hóa đơn điện tử của doanh nghiệp tại Chi cục thuế quận Tân Phú - TP.HCM có ý nghĩa khoa học và thực tiễn. Kết quả nghiên cứu sẽ giúp cho Chi Cục Thuế quận Tân Phú đưa ra những phương án để $100 \% \mathrm{DN}$ trên địa bàn sử dụng hóa đơn điện tử

\section{CƠ SỞ LÝ THUYẾT}

2.1 Tổng quan về quản lý thuế và hóa đơn điện tử

Quản lý thuế là một quy trình được xây dựng một cách chặt chẽ. Việc quyết toán thuế bắt đầu từ khâu xác định đối tượng nộp thuế, lập hồ sơ thuế và thông báo thuế, tổ chức thu nộp tại cơ quan kho bạc. Mỗi đối tượng quản lý thuế, chẳng hạn đối tượng thực hiện theo phương pháp khấu trừ, phương pháp trực tiếp trên 
doanh thu hay khoán thuế hoặc theo từng sắc thuế: thuế giá trị gia tăng, thuế thu nhập doanh nghiệp, thuế thu nhập cá nhân... đều phải xây dựng một quy trình cụ thể, chặt chẽ theo nguyên tắc khép kín, có sự độc lập của từng khâu, kiểm tra, giám sát lẫn nhau. Cơ sở để quyết toán thuế là các chứng từ thể hiện trong hoạt động kinh doanh của đối tượng khao báo thuế. Trong đó, hóa đơn tài chính là một chứng từ quan trọng không thể thiếu.

Hóa đơn, ngày nay tùy theo $\mathrm{DN}$, cỏ thể sử dụng hóa đơn truyền thống hoặc hóa đơn điện tử Hóa đơn truyền thống là chứng từ kế toán do tổ chức, cá nhân bán hàng hóa, cung cấp dịch vụ lập, ghi nhận thông tin bán hàng hóa, cung cấp dịch vụ theo quy định của luật kế toán (Nghị định

119/2018/NĐ-CP).

Nhược điểm của hình thức giao hóa đơn này là thời gian và chi phí. Khi hóa đơn giấy được giao tay, phải tốn nhiều lao động và thường hay bị lỗi, nó hay gây ra chậm trễ và các sai sót không ngờ tới. Khi các trường hợp trên xảy ra, kết quả doanh nghiệp phải chịu chi phí cao hơn. Bên cạnh đó, phải mất thời gian để một hóa đơn giấy đến đích của nó.

Theo Thông tư 32/2011/TT-BTC, hoá đơn điện tử là tập hợp các thông điệp dữ liệu điện tử về bán hàng hoá, cung ứng dịch vụ, được khởi tạo, lập, gửi, nhận, lưu trữ và quản lý bằng phương tiện điện tử. Hoá đơn điện tử được khởi tạo, lập, xử lý trên hệ thống máy tính của tổ chức đã được cấp mã số thuế khi bán hàng hoá, dịch vụ và được lưu trữ trên máy tính của các bên theo quy định của pháp luật về giao dịch điện tử.

Hóa đơn điện tử đảm bảo nguyên tắc: xác định được số hóa đơn theo nguyên tắc liên tục và trình tự thời gian, mỗi số hóa đơn đảm bảo chỉ được lập và sử dụng một lần duy nhất.

Hóa đơn đã lập dưới dạng giấy nhưng được xử lý, truyền hoặc lưu trữ bằng phương tiện điện tử không phải là hóa đơn điện tử.

Hóa đơn điện tử có giá trị pháp lý nếu thỏa mãn đồng thời các điều kiện. Thứ nhất có sự đảm bảo đủ tin cậy về tính toàn vẹn của thông tin chứa trong hóa đơn điện tử từ khi thông tin được tạo ra ở dạng cuối cùng là hóa đơn điện tử. Thứ hai, tiêu chí đánh giá tính toàn vẹn là thông tin còn đầy đủ và chưa bị thay đổi, ngoài những thay đổi về hình thức phát sinh trong quá trình trao đổi, lưu trữ hoặc hiển thị hóa đơn điện tử. Thứ ba, thông tin chứa trong hóa đơn điện tử có thể truy cập, sử dụng được dưới dạng hoàn chỉnh khi cần thiết.

\section{Lợi ích của việc sử dung hóa đơn điện tủ?}

Cùng với sự phát triển chung của cuộc cách mạng công nghệ 4.0, HĐĐT đang dần được sử dụng để thay thế hóa đơn giấy thông thường. Việc sử dụng phẩn mềm hóa đơn điện tử mang lại rất nhiều lợi ích thiêt thực giúp doanh nghiệp tiết kiệm được các chi phí in ấn, gửi hóa đơn và quản lý hóa đơn tiện lợi hơn. Cụ thể là tiết kiệm chi phí vận chuyển, bảo quản hóa đơn, an toàn; Bảo mật; Tiết kiệm thời gian, giảm thiểu các thủ tục hành chính; Không cần phải lập báo cáo tình hình sử dụng hóa đơn; Tiện ích cao, đa dạng phương thức gửi hóa đơn cho khách hàng

Với tiện ích truy xuất online, khách hàng doanh nghiệp có thể sử dụng nhiều HĐĐT tùy chọn theo nhu cầu tại đơn vị với phương thức lưu trữ đa dạng như gửi trực tiếp cho người bán theo cách thức truyền nhận HĐĐT đã thỏa thuận giữa hai bên như qua Email, SMS.

\subsection{Lý thuyết về ý định hành vi}

Ý định hành vi bị ảnh hưởng bởi hai yếu tố: thái độ và chuẩn chủ quan. Sau đó, Davis (1989) đưa ra mô hình chấp nhận công nghệ TAM (Technology Acceptance Model) để giải thích các yếu tố ảnh hưởng sự chấp nhận công nghệ và hành vi người sử dụng công nghệ trên cơ sở của lý thuyết TRA (Theory of Reasoned Action). Mô hình TAM khảo sát mối liên hệ và ảnh hưởng của các yếu tố nhận thức tính dễ sử dụng, nhận thức sự hữu ích đến thái độ, từ đó ảnh hưởng đến ý định và hành vi trong việc chấp nhận công nghệ thông tin của người sử dụng. Ý định được xem là tiền đề trực tiếp dẫn đến hành vi sử dụng công nghệ trong mô hình TAM. Nghiên cứu của Zhang và ctg (2010) cũng khẳng định ý định sử dụng là một khái niệm rất quan trọng trong nghiên cứu hành vi tiêu dùng và cũng là yếu tố quan trọng nhất quyết định hành vi tiêu dùng thực tế.

Thuyết TPB được Ajzen xây dựng bằng cách bổ sung thêm biến "Hành vi kiểm soát cảm nhận" vào mô hình TRA. Biến này bị tác động bởi hai biến số là niềm tin kiểm soát và sự dễ dàng cảm nhận. Niềm tin kiểm soát được định nghĩa là một cá nhân cảm thấy tự tin về khả năng của anh/cô ta để thực hiện một hành vi, tương tự như sự tự tin. Kết quả mô hình TPB thì từ hành vi dự định sẽ hướng đến hành vi thực sự. Ngoài ra, việc kết hợp hai mô hình TAM và TPB trong cùng lĩnh vực (domain) sẽ tạo ra sức mạnh trong việc dự đoán tốt hơn là sử dụng riêng lẻ mô hình $\mathrm{TAM}$ hoặc $\mathrm{TPB}$. Đối với các nghiên cứu có sử dụng sản phẩm 
công nghệ thì việc tăng thêm các yếu tố về công nghệ cho TAM (kết hợp thuyết hành vi dự định TPB) trong mô hình sẽ thích hợp hơn. Ví dụ như mô hình C-TAM-TPB được dùng để dự đoán xu hướng sử dụng của đối tượng chưa sử dụng công nghệ trước đây; tương tự như việc dự đoán thói quen sử dụng của đối tượng đã sử dụng hoặc có quen thuộc với công nghệ.

\subsection{Mô hình nghiên cứu}

Nghiên cứu của Harald, B. (2009) cho thấy trong báo cáo của mình rằng hệ thống HĐĐT là một mô hình ba chân thể hiện nền tảng cải thiện việc sử dụng hệ thống cải tiến này. Mô hình này bao gồm ba khối công việc, bao gồm xây dựng các yêu cầu pháp lý để hướng dẫn các doanh nghiệp thực hiện khi sử dụng HĐĐT, khả năng tương tác giữa các nhà vận hành và nội dung hướng dẫn tiêu chuẩn. Mô hình này được phát triển dựa trên một bộ các yêu cầu kinh doanh và thực hiện theo đề nghị và liên lạc để gia tăng tích hợp hệ thống HĐĐT này (Harald 2009, 19.)

Nghiên cứu của Nguyễn Thị Hồng Liêm (2016) về "Các yếu tố ảnh hưởng đến xu hướng chọn hóa đơn điện tử của doanh nghiệp". Dựa trên cơ sở lý thuyết và các nghiên cứu trước, tác giả đề xuất mô hình nghiên cứu cho đề tài, được hình thành dựa trên hai mô hình đó là (1) Mô hình kết hợp TAM và TPB (1995) đã được nhiều nghiên cứu trước ứng dụng. Tác giả đề xuất 6 yếu tố chính tác động đến xu hướng chọn hóa đơn điện tử của người doanh nghiệp là (1) Nhận thức sự hữu ích, (2) Nhận thức tính dễ sử dụng, (3) Chuẩn chủ quan, (4) Nhận thức kiểm soát hành vi, (5) Niềm tin và (6) Nhận thức về rào cản chuyển đồi. Kết quả nghiên cứu cho thấy có 6 yếu tố ảnh hưởng tích cực (cùng chiều) đển xu hướng chọn hóa đơn điện tử theo mức độ từ cao tới thấp là: nhận thức kiểm soát hành vi, nhận thức sự hữu ích, nhận thức tính dễ sử dụng, niềm tin, chuẩn chủ quan và cuối cùng là nhận thức rào cản chuyển đổi

Nghiên cứu của Lê Thị Kim Tuyến (2011) dựa trên cơ sở lý thuyết xuất phát từ các mô hình TRA, TBP, TAM, IDT và kết quả của nghiên cứu định tính, tác giả đề xuất mô hình động cơ sử dụng dịch vụ Internet Banking của người tiêu dùng tại Thành phố Đà Nẵng. Kết quả cuối cùng cho thấy có 8 yếu tố ảnh hưởng đến động cơ khiến khách hàng sử dụng Internet Banking là: Nhận thức hữu ích; Hiểu biết; Tính tương hợp; Rủi ro; Ẩnh hưởng xã hội; Tính linh động; Phong cách; Công việc.

Dựa trên cơ sở lý thuyêt ý định hành vi và các nghiên cứu trước trên trên nền tảng: Thuyết hành động hợp lý (TRA); Thuyết hành vi hoạch định (TPB); Mô hình chấp nhận công nghệ (TAM), tác giả đề xuất mô hình nghiên cứu "Các yếu tố ảnh hưởng đến ý định sử dụng hóa đơn điện tử của doanh nghiệp tại Chi cục Thuế quận Tân Phú - TP.HCM" bao gồm 6 nhân tố là (1) Sự hữu ích, (2) Dễ sử dụng, (3) Sự tin tưởng, (4) Chi phí, (5) Sự tương hỗ và (6) Chuẩn chủ quan.

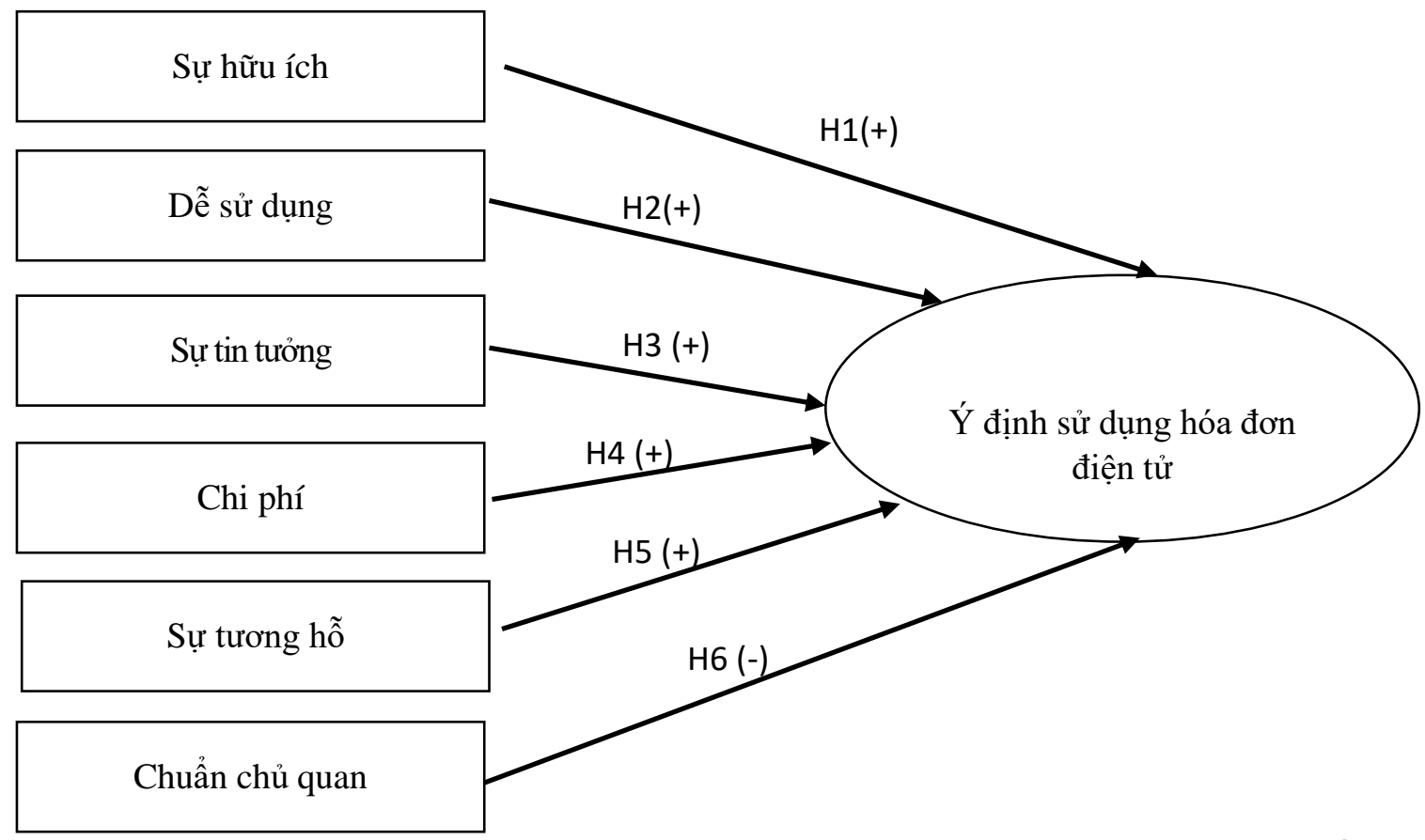

Nguồn: Tác giả

Hình 1 Mô hình nghiên cứu đề xuất của tác giả 


\section{PHƯƠNG PHÁP NGHIÊN CÚU}

Tác giả sử dụng phương pháp nghiên cứu hỗn hợp định tính và định lượng. Nghiên cứu định tính được tác giả sử dụng để phỏng vấn sâu 4 chuyên gia và thảo luận với 6 thành viên để xác định mô hình nghiên cứu và thang đo. Phương pháp nghiên cứu định lượng trãi qua 2 giai đoạn. Giai đoạn 1 khảo sát sơ bộ 30 phiếu để sàng lọc, xử lý, hoàn chỉnh bảng câu hỏi, tiến hành khảo sát chính thức.

Phương pháp lấy mẫu của tác giả là phi xác suất, thuận tiện. Tác giả phát 250 phiếu khảo sát trực tiếp cho các doanh nghiệp khi lên Chi Cục Thuế Tân Phú làm thủ tục quyết toán thuế. Việc phát phiếu này có tính chất ngẫu nhiên và tạo điều kiên thuận tiện cho tác giả thu thập thông tin, dữ liệu sơ cấp. Kích cỡ mẫu, tác giả áp dụng theo Hair etal (2006) với $\mathrm{n} \geq 5^{*}$ tổng số biến quan sát. Trong bảng câu hỏi có 6 biến độc lập với 28 biến quan sát, như vậy $\mathrm{n} \geq 5^{*} 28=140$. Để đảm bảo độ tin cậy cho nghiên cứu, tác giả phát ra 250 phiếu và thu hồi về được 235 phiếu hợp lệ đưa vào kiểm định, phân tích bằng phần mềm SPSS 20.0

\section{KẾT QUẢ NGHIÊN CÚ̉ VÀ THẢO LUẬN \\ 4.1 Kiểm định độ tin cậy của thang đo}

Bảng 1: Kiểm định độ tin cậy biến độc lập và biến phụ thuộc

\begin{tabular}{|c|c|c|c|c|}
\hline $\begin{array}{c}\text { Biến } \\
\text { quan sát }\end{array}$ & $\begin{array}{l}\text { Trung bình } \\
\text { thang đo nếu } \\
\text { loại biến }\end{array}$ & $\begin{array}{l}\text { Phương sai } \\
\text { thang đo nếu } \\
\text { loại biến }\end{array}$ & $\begin{array}{c}\text { Tương quan } \\
\text { với biến tổng }\end{array}$ & $\begin{array}{l}\text { Cronbach's } \\
\text { alpha nếu } \\
\text { loại biến }\end{array}$ \\
\hline \multicolumn{5}{|c|}{ Độ tin cậy của thang đo sự hữu ích (HI): Cronbach's Alpha = 0.876} \\
\hline HI2 & 9.58 & 9.117 & .771 & .826 \\
\hline HI3 & 8.94 & 8.718 & .669 & .874 \\
\hline HI5 & 9.66 & 9.747 & .740 & .841 \\
\hline HI6 & 9.57 & 9.100 & .776 & .824 \\
\hline \multicolumn{5}{|c|}{ Độ tin cậy của thang đo dễ sử dụng $(\mathrm{SD})$ : Cronbach's Alpha = 0.915} \\
\hline SD1 & 10.93 & 11.785 & .816 & .886 \\
\hline SD2 & 10.98 & 12.106 & .813 & .887 \\
\hline SD3 & 10.95 & 11.720 & .798 & .892 \\
\hline SD4 & 10.99 & 11.949 & .794 & .893 \\
\hline \multicolumn{5}{|c|}{ Độ tin cậy của thang đo sự tin tưởng (STT): Cronbach's Alpha $=0.757$} \\
\hline STT1 & 13.93 & 14.363 & .633 & .672 \\
\hline STT2 & 13.91 & 14.427 & .584 & .691 \\
\hline STT3 & 13.80 & 15.252 & .610 & .684 \\
\hline STT4 & 13.94 & 14.266 & .685 & .653 \\
\hline STT5 & 13.45 & 20.266 & .139 & .825 \\
\hline \multicolumn{5}{|c|}{ Độ tin cậy của thang đo sự tin tưởng (lần 2): Cronbach's Alpha $=0.825$} \\
\hline STT1 & 10.12 & 11.835 & .661 & .775 \\
\hline STT2 & 10.10 & 11.781 & .622 & .794 \\
\hline STT3 & 10.00 & 12.620 & .644 & .784 \\
\hline STT4 & 10.14 & 12.016 & .679 & .767 \\
\hline \multicolumn{5}{|c|}{ Độ tin cậy của thang đo chi phí (CP): Cronbach's Alpha = 0.883} \\
\hline $\mathrm{CP} 1$ & 10.23 & 13.175 & 689 & .871 \\
\hline $\mathrm{CP} 2$ & 10.11 & 12.606 & .737 & .853 \\
\hline CP3 & 10.10 & 12.405 & .762 & .843 \\
\hline $\mathrm{CP} 4$ & 9.93 & 12.469 & .796 & .830 \\
\hline \multicolumn{5}{|c|}{ Độ tin cậy của thang đo sự tương hỗ $(\mathrm{TH})$ : Cronbach's Alpha $=0.865$} \\
\hline TH1 & 10.56 & 9.590 & .719 & .825 \\
\hline TH2 & 10.52 & 9.644 & .747 & .814 \\
\hline TH3 & 10.37 & 9.421 & .673 & .846 \\
\hline TH4 & 10.35 & 9.845 & .721 & .824 \\
\hline \multicolumn{5}{|c|}{ Độ tin cậy của thang đo chuẩn chủ quan (CQ): Cronbach's Alpha = 0.832} \\
\hline CQ1 & 13.25 & 22.103 & .652 & .793 \\
\hline
\end{tabular}



TẠI CHI CỤC THUÊ QUẬN TÂN PHÚ - TP.HCM

\begin{tabular}{|c|c|c|c|c|}
\hline CQ2 & 13.26 & 21.612 & .676 & .786 \\
\hline CQ3 & 13.28 & 22.374 & .648 & .794 \\
\hline CQ4 & 13.17 & 23.045 & .560 & .819 \\
\hline CQ5 & 13.20 & 22.522 & .621 & .802 \\
\hline \multicolumn{5}{|c|}{ Độ tin cậy của thang đo ý định (YD): Cronbach's Alpha = 0.853} \\
\hline YD1 & 17.94 & 21.872 & .690 & .819 \\
\hline YD2 & 17.94 & 22.574 & .598 & .835 \\
\hline YD3 & 18.01 & 22.141 & .602 & .835 \\
\hline YD4 & 18.00 & 22.274 & .666 & .823 \\
\hline YD5 & 17.98 & 22.500 & .616 & .832 \\
\hline YD6 & 17.98 & 21.534 & .661 & .824 \\
\hline
\end{tabular}

Nguồn: Hệ thống phần mềm SPSS

Kết quả chạy phân tích độ tin cậy của thang đo sự hữu ích lần 3 cho thấy độ tin cậy của thang đo bằng 0,876 $>0,6$ đạt yêu cầu. Tất cả các biến thành phần đều có tương quan với tổng lớn hơn 0.3 . Như vậy thang đo nhân tố HI với các biến quan sát: HI2, HI3, HI5, HI6 đạt độ tin cậy. Kết quả chạy phân tích độ tin cậy của thang đo sự tin tưởng 2 lần cho thấy độ tin cậy của thang đo bằng $0,825>0,6$ đạt yêu cầu. Tất cả các biến thành phần đều có tương quan với tổng lớn hơn 0.3 . Như vậy thang đo nhân tố STT với các biến quan sát: STT1, STT2, STT3, STT4 đạt độ tin cậy, biến STT5 bị loại. Ngoài ra, các nhân tố khác như SD, CP, TH, $\mathrm{CQ}$ và biến phụ thuộc $\mathrm{YD}$ đều có độ tin cậy của thang đo $>0,6$ đạt yêu cầu. Các biến thành phần đều có tương quan với tổng lớn hơn 0.3 . Như vậy các biến này đạt yêu cầu để đưa vào phân tích nhân tố khám phá

\subsection{Phân tích EFA}

\section{Phân tích nhân tố các thành phần thang đo}

Kết quả phân tích EFA các nhân tố (biến độc lập) ảnh hưởng đến ý định sử dụng hóa đơn điện tử của doanh nghiệp được thể hiện chi tiết trong bảng sau:

Bảng 2: Kết quả phân tích EFA cho các biến độc lập

\begin{tabular}{|c|c|c|c|c|c|c|}
\hline \multirow{2}{*}{ Biến quan sát } & \multicolumn{6}{|c|}{ Hệ số tải nhân tố của các thành phần } \\
\hline & 1 & 2 & 3 & 4 & 5 & 6 \\
\hline SD1 & .872 & & & & & \\
\hline SD2 & .859 & & & & & \\
\hline SD4 & .838 & & & & & \\
\hline SD3 & .834 & & & & & \\
\hline $\mathrm{CP} 4$ & & .864 & & & & \\
\hline CP3 & & .825 & & & & \\
\hline $\mathrm{CP} 2$ & & .804 & & & & \\
\hline $\mathrm{CP} 1$ & & .741 & & & & \\
\hline HI6 & & & .857 & & & \\
\hline $\mathrm{HI} 2$ & & & .843 & & & \\
\hline HI5 & & & .801 & & & \\
\hline $\mathrm{HI} 3$ & & & .718 & & & \\
\hline CQ2 & & & & .758 & & \\
\hline CQ3 & & & & .725 & & \\
\hline CQ1 & & & & .721 & & \\
\hline CQ5 & & & & .703 & & \\
\hline CQ4 & & & & .621 & & \\
\hline TH2 & & & & & .814 & \\
\hline TH1 & & & & & .798 & \\
\hline TH4 & & & & & .783 & \\
\hline TH3 & & & & & .721 & \\
\hline STT4 & & & & & & .765 \\
\hline STT1 & & & & & & .764 \\
\hline
\end{tabular}




\begin{tabular}{|c|c|c|c|c|c|c|}
\hline \multirow{2}{*}{ Biến quan sát } & \multicolumn{6}{|c|}{ Hệ số tải nhân tố của các thành phần } \\
\hline & $\mathbf{1}$ & 2 & 3 & 4 & 5 & 6 \\
\hline STT3 & & & & & & .727 \\
\hline STT2 & & & & & & 699 \\
\hline Eigenvanlues & 3.996 & 3.166 & 3.014 & 2.75 & 2.587 & 2.304 \\
\hline Phương sai trích (\%) & 14.60 & 25.92 & 36.67 & 45.95 & 54.76 & 62.46 \\
\hline Cronbach's Cronbach's Alpha & 0.915 & 0.883 & 0.876 & 0.832 & 0.865 & 0.825 \\
\hline Sig. & & & & & & 0.000 \\
\hline KMO & & & & & & 0.783 \\
\hline Tổng Phương sai trích (\%) & & & & & & $2.47 \%$ \\
\hline
\end{tabular}

\section{Phân tích EFA cho biến phụ thuộc}

Nguồn: Hệ thống phần mềm SPSS

Thực hiện phân tích EFA theo phương pháp trích yếu tố Principal components với phép quay Varimax, Kết quả thể hiện trong bảng sau:

Bảng 3: Kết quả phân tích EFA cho biến phụ thuộc

\begin{tabular}{|c|c|}
\hline \multicolumn{1}{|c|}{ Biến quan sát } & Ý định sử dụng \\
\hline YD1 & .761 \\
\hline YD4 & .735 \\
\hline YD6 & .727 \\
\hline YD5 & .676 \\
\hline YD3 & .659 \\
\hline YD2 & .653 \\
\hline Eigenvanlues & 3.465 \\
\hline Phương sai trích (\%) & 57.75 \\
\hline Cronbach's Cronbach's Alpha & 0.835 \\
\hline Sig. & 0.000 \\
\hline KMO & 0.886 \\
\hline
\end{tabular}

\section{Phân tích EFA}

Nguồn: Hệ thống phần mềm SPSS

Như vậy, sau khi thực hiện kiểm định EFA cho biến độc lập và biến phụ thuộc. Kết quả phân tích nhân tố cho thấy các biến độc lập và biến phụ thuộc trong mô hình đều đạt giá trị hội tụ và giá trị phân biệt chấp nhận được: phân tích EFA là thích hợp với dữ liệu nghiên cứu. Có 06 nhân tố được trích ra từ kết quả phân tích bao gồm 25 biến quan sát. Tất cả các biến quan sát trong từng nhân tố tương ứng được trích đều đạt yêu cầu và được sử dụng trong các phân tích tiếp theo. Do vậy không hiệu chỉnh thang đo và mô hình nghiên cứu đã đề xuất ban đầu.

\subsection{Kiểm định mô hình và các giả thuyết}

\subsubsection{Phân tích tương quan}

Kết quả phân tích tương quan có 05 các biến độc lập đều có tương quan với biến phụ thuộc ở mức ý nghĩa $5 \%$. Tuy nhiên, biến độc lập GC có Sig $=0.104>5 \%$, do đó biến CP không có sự tương quan tuyến tính với biến phụ thuộc YD. Biến phụ thuộc YD có tương quan mạnh nhất với biển độc lập HI (hệ số Pearson = 0.326 ) và tương quan yếu nhất với biến độc lập CQ (hệ số Pearson $=0.287$ ). Sự tương quan chặt này rất được mong đợi vì chính những mối quan hệ chặt, tuyến tính giữa các biến giải thích được sự ảnh hưởng đến kết quả mô hình. Do đó, các biến độc lập này có thể đưa vào phân tích hồi quy để giải thích ảnh hưởng đến kết quả của mô hình nghiên cứu.

Bảng 4: Kết quả tương quan Pearson

\begin{tabular}{|c|c|c|c|c|c|c|c|c|}
\hline \multicolumn{9}{|c|}{ Hệ số tương quan } \\
\hline & & YD_TB & HI_TB & SD_TB & STT_TB & CP_TB & TH_TB & CQ_TB \\
\hline \multirow{3}{*}{ YD_TB } & Pearson Correlation & 1 & $.326^{\text {*** }}$ & $.302^{* * *}$ & $243^{* * *}$ & .106 & $.300^{* *}$ & $.287^{* * *}$ \\
\hline & Sig. (2-tailed) & & .000 & .000 & .000 & .104 & .000 & .000 \\
\hline & $\mathrm{N}$ & 235 & 235 & 235 & 235 & 235 & 235 & 235 \\
\hline
\end{tabular}



TẠI CHI CỤC THUÊ QUẬN TÂN PHÚ - TP.HCM

\begin{tabular}{|c|c|c|c|c|c|c|c|c|}
\hline \multicolumn{9}{|c|}{ Hê số tương quan } \\
\hline \multirow{3}{*}{ HI_TB } & Pearson Correlation & $.326^{* *}$ & 1 & .032 & -.010 & -.031 & .105 & .067 \\
\hline & Sig. (2-tailed) & .000 & & .628 & .882 & .638 & .108 & .308 \\
\hline & $\mathrm{N}$ & 235 & 235 & 235 & 235 & 235 & 235 & 235 \\
\hline \multirow{3}{*}{ SD_TB } & Pearson Correlation & $.302^{* * *}$ & .032 & 1 & .030 & .008 & $.155^{*}$ & .065 \\
\hline & Sig. (2-tailed) & .000 & .628 & & .642 & .904 & .017 & .320 \\
\hline & $\mathrm{N}$ & 235 & 235 & 235 & 235 & 235 & 235 & 235 \\
\hline \multirow{3}{*}{ STT_TB } & Pearson Correlation & $.243^{* * *}$ & -.010 & .030 & 1 & .060 & .113 & .016 \\
\hline & Sig. (2-tailed) & .000 & .882 & .642 & & .362 & .085 & .813 \\
\hline & $\mathrm{N}$ & 235 & 235 & 235 & 235 & 235 & 235 & 235 \\
\hline \multirow{3}{*}{ CP_TB } & Pearson Correlation & .106 & -.031 & .008 & .060 & 1 & .099 & .026 \\
\hline & Sig. (2-tailed) & .104 & .638 & .904 & .362 & & .128 & .697 \\
\hline & $\mathrm{N}$ & 235 & 235 & 235 & 235 & 235 & 235 & 235 \\
\hline \multirow{3}{*}{ TH_TB } & Pearson Correlation & $.300^{* * *}$ & .105 & $.155^{*}$ & .113 & .099 & 1 & .111 \\
\hline & Sig. (2-tailed) & .000 & .108 & .017 & .085 & .128 & & .089 \\
\hline & $\mathrm{N}$ & 235 & 235 & 235 & 235 & 235 & 235 & 235 \\
\hline \multirow{3}{*}{ CQ_TB } & Pearson Correlation & $.287^{* * *}$ & .067 & .065 & .016 & .026 & .111 & 1 \\
\hline & Sig. (2-tailed) & .000 & .308 & .320 & .813 & .697 & .089 & \\
\hline & $\mathrm{N}$ & 235 & 235 & 235 & 235 & 235 & 235 & 235 \\
\hline
\end{tabular}

\subsubsection{Phân tích hồi quy đa biến}

Nguồn : Xử lý SPSS

Phân tích hồi quy được thực hiện với 6 biến độc lập và phương pháp chọn là "Enter". Kết quả phân tích hồi quy đa biến của mô hình cho thấy $\mathrm{R}^{2}$ hiệu chỉnh là 0.609 , có nghĩa là $60.1 \%$ sự biến thiên của biến phụ thuộc YD được giải thích chung bởi các biến độc lập trong mô hình. Bên cạnh đó, kiểm định $\mathrm{F}$ cũng cho thấy giá trị Sig. rất nhỏ $(\mathrm{Sig} .=.000)$, cho thấy mô hình trên phù hợp với tập dữ liệu đang khảo sát. Các biến độc lập HI, SD, STT, TH, CQ đều có ý nghĩa về mặt thống kê (Sig. < 0.05 ); biến phụ thuộc GC có Sig. = $0.153>5 \%=>$ Biến phụ độc lập GC không có ý nghĩa thống kê với mức ý nghĩa $5 \%$ đến Ý định sử dụng HĐĐT của Doanh nghiệp. Kết quả cho thấy hệ số chấp nhận (Tolerance) khá cao (từ 0.937 đến 0.986 ) và hệ số phóng đại phương sai VIF thấp (từ 1.014 đến $1.068<2$ ). Do vậy, có thể kết luận mối liên hệ giữa các biến độc lập này không đáng kể, không có hiện tượng đa cộng tuyến.

Bảng 4: Kết quả hồi quy đa biến

\begin{tabular}{|c|c|c|c|c|c|c|c|}
\hline \multirow{2}{*}{ Model } & \multicolumn{2}{|c|}{$\begin{array}{l}\text { Unstandardized } \\
\text { Coefficients }\end{array}$} & \multirow{2}{*}{$\begin{array}{c}\text { Standardized } \\
\text { Coefficients } \\
\text { Beta }\end{array}$} & \multirow[t]{2}{*}{$\mathrm{t}$} & \multirow{2}{*}{ Sig. } & \multicolumn{2}{|c|}{ Collinearity Statistics } \\
\hline & $\mathrm{B}$ & Std. Error & & & & Tolerance & VIF \\
\hline (Constant) & .064 & .338 & & .189 & .850 & & \\
\hline HI_TB & .266 & .050 & .289 & 5.339 & .000 & .984 & 1.017 \\
\hline SD_TB & .199 & .044 & .243 & 4.476 & .000 & .973 & 1.028 \\
\hline STT_TB & .174 & .045 & .211 & 3.904 & .000 & .984 & 1.016 \\
\hline CP_TB & .062 & .043 & .077 & 1.433 & .153 & .986 & 1.014 \\
\hline TH_TB & .161 & .051 & .175 & 3.162 & .002 & .937 & 1.068 \\
\hline CQ_TB & .182 & .043 & .227 & 4.194 & .000 & .982 & 1.018 \\
\hline
\end{tabular}

a. Dependent Variable: YD_TB

Nguồn: Hệ thống phần mềm SPSS

Dựa vào kết quả hồi quy, hệ số Beta sau khi đã loại biến GC. Ta có thể viết phương trình hồi quy bội biểu diễn mối quan hệ giữa các nhân tố và ý định sử dụng hóa đơn điện tử của các doanh nghiệp tại Chi cục thuế quận Tân Phú - TP.HCM như sau:

Trong đó:

$$
Y=0.64+0.289 X_{1}+0.243 X_{2}+0.211 X_{3}+0.175 X_{4}+0.227 X_{5}
$$

- Y là biến phụ thuộc thể hiện ý định sử dụng hóa đơn điện tử 
- X1, X2, X3, X4, X5 là các biến độc lập theo thứ tự là: Sự hữu ích, dễ sử dụng, sự thuận tiện, sự tương hỗ và chuẩn chủ quan.

Căn cứ vào kết quả phân tích hồi quy, mục tiêu thứ nhất của đề tài đã được trả lời: có 05 yếu tố có ảnh hưởng đến Ý định sử dụng hóa đơn điện tử của các doanh nghiệp tại Chi cục thuế quận Tân Phú - TP.HCM: (1) Sự hữu ích, (2) Dễ sử dụng, (3) Sự thuận tiện, (4) Sự tương hổ, và (5) Chuẩn chủ quan.

Trong 05 yếu tố trên, yếu tố "sự hữu ích" có tác động lớn nhẩt đến ý định sử dụng HĐĐT của DN với hệ số hồi quy là 0.289 , tiếp đến là yếu tố "dễ sử dụng" có hệ số hồi quy là 0.243 , yếu tố " sự thuận tiện" có tác động thấp hơn với hệ số hồi quy 0.211 , yếu tố "chuẩn chủ quan" với hệ số hồi quy là 0.223 , yếu tố và yếu tố "sự tương hỗ" (nhân tố mới trong nghiên cứu của tác giả) với hệ số hồi quy thấp nhất là 0.175 .

\subsubsection{Kiểm định về tính độc lập của sai số trong mô hình, hiện tự̣ng đa cộng tuyến}

Từ Giá trị Durbin - Watson cho thấy $\mathrm{D}=1.1771$, giá trị $\mathrm{D}$ nằm trong miền chấp nhận cho thấy mô hình không có tự tương quan giữa các phần dư.

Từ chỉ số VIF cho thấy các chỉ số VIF đều nhỏ hơn 10 nên không có hiện tượng đa cộng tuyến giữa các biến độc lập (Hoàng Trọng \& Mộng Ngọc, 2005).

\subsubsection{Kiểm định các giả thuyết}

Kết quả kiểm định các giả thuyết thì kết quả ước lượng cho thấy mối quan hệ giữa các nhân tố (HI), (DSD), $(\mathrm{TT}),(\mathrm{TH}),(\mathrm{CQ})$ và ý định sử dụng HĐĐT đều $>0$ ở mức ý nghĩa thống kê Sig. $<0,05$, mặt khác hệ số Beta của các nhân tố này đều > 0 nên các giả thuyết $\mathrm{H1}, \mathrm{H} 2, \mathrm{H} 3, \mathrm{H} 5, \mathrm{H} 6$ được chấp nhận với mẫu dữ liệu khảo sát. Như vậy, những yếu tố này đều có ảnh hưởng đến ý định sử dụng HĐĐT,

Riêng chỉ có kêt quả ước lượng cho thấy, có sự tương quan thuận giữa chi phí và ý định sử dụng hóa đơn điện tử. Tuy nhiên, hệ số tương quan giữa "chi phî" và "ý định sử dụng hóa đơn điện tử” là 0.077 với mức ý nghĩa Sig. $=0.153>0.05$ (bảng 4) do đó giả thuyết H4 bị bác bỏ. Như vậy, chi phí HĐĐT không có ý nghĩa thống kê trong việc tác động đến ý định sử dụng hóa đơn điện tử của doanh nghiệp với mức ý nghĩa $5 \%$.

\subsubsection{Kiểm định Independent Sample T-Test và Anova}

Kết quả kiểm định cho thấy:

- Kiểm định Independent Sample T-Test cho kết quả Levene's Test có giá trị Sig. $=0.952$ ta sử dụng kết quả tại cột T-test for Equality of Means có kết quả Sig $=0.955>0.5 \Rightarrow$ Không có sự khác biệt về ý định sử dụng hóa đơn điện tử giữa những người trả lời có giới tính khác nhau => Bác bỏ giả thuyết H1.

Thực hiện kiểm định ANOVA cho các giả thuyết H2, H3, H4 kết quả cho thấy

- Giả thuyết H2 (sig. của test of Homogeneity of Variances: 0.581, sig. Anova: 0.646) nên không có sự khác biệt về ý định sử dụng hóa đơn điện tử đối với những người có độ tuồi khác nhau => Bác bỏ giả thuyết $\mathrm{H} 2$

- Giả thuyết H3 (sig. của test of Homogeneity of Variances: $0.03<0.05$, ta không sử dụng kết quả sig. của bảng Anova mà sử dụng kết quả từ bảng kết quả kiểm định Welch. Kết quả từ bảng kiểm định Welch cho thấy Sig. $=0.273>0.05=>$ Không có sự khác biệt về ý định sử dụng hóa đơn điện tử giữa những người trả lời có chức vụ khác nhau => Bác bỏ giả thuyết $\mathrm{H} 3$.

- Giả thuyết H4 (sig. của test of Homogeneity of Variances: 0.943, sig. Anova: 0.826) nên không có sự khác biệt về ý định sử dụng hóa đơn điện tử đối với những người có thâm niên khác nhau $=>$ Bác bỏ giả thuyết $\mathrm{H} 4$

- Kết quả kiểm định phương sai cho thấy mức ý nghĩa (sig. của test of Homogeneity of Variances: 0.241, sig. Anova: 0.597) nên không có sự khác biệt về ý định sử dụng hóa đơn điện tử đối với những nhóm loại hình doanh nghiệp khác nhau $=>$ Bác bỏ giả thuyết $\mathrm{H}_{0}$.

Như vậy, không có sự khác biệt về ý định sử dụng hóa đơn điện tử giữa các người trả lời có giới tính, chức vụ, thâm niên làm việc và loại hình doanh nghiệp khác nhau.

\subsection{Thảo luận}

Mô hình nghiên cứu tác giả đề xuất có 6 biến độc lập với 28 biến quan sát. Qua kiểm định độ tin cậy Cronbach's Alpha và phân tích EFA có 3 biến bị loại, còn lại 25 biến quan sát. Khi kiểm định hồi qui thì yếu tố chi phí bị loại. Trong thực tế chi phí bị loại khá phù hợp vì các doanh nghiệp tất yếu là phải đầu tư chi phí vào phần mềm kế toán trong đó có khâu xuất HĐĐT phục vụ cho việc thu, chi trong giao dịch và quyết toán kinh doanh cuối kỳ. Như vậy, kết quả có 5 yếu tố ảnh hưởng đến ý định sử dụng HĐĐT của 
doanh nghiệp theo thứ tự mức độ ảnh hưởng là sự hữu ích, dễ sử dụng, sự thuận tiện, sự tương hỗ và chuẩn chủ quan

Ngoài ra, kết quả kiểm định Independent Sample T-Test và Anova cũng khẳng định không có cơ sở kết luận có sự khác biệt giữa ý định sử dụng hóa đơn điện tử với những nhóm người khác nhau về độ tuổi, giới tính, thời gian làm việc, chức vụ, thâm niên công tác, loại hình doanh nghiệp.

\section{HÀM Ý QUẢN TRI}

\section{Sư hứu ích của hóa đơn điện tủ̉}

Xây dựng nhận thức và tầm ảnh hưởng của HĐĐT cho từng nhân viên đang công tác tại doanh nghiệp để họ có những động thái tích cực chuẩn bị cho những thay đổi và bắt kịp với xu thế mới. Doanh nghiệp cần thường xuyên tổ chức các buổi họp, hội thảo nhằm giới thiệu, đánh giá những mặt tích cực và hạn chế trong quá trình sử dụng, khai thác HĐĐT để có những điều chỉnh kịp thời.

\section{Hoàn thiện nâng cao chất lựng phần mềm hóa đơn điện tủ̉, đảm bảo tính dễ sử dụng}

Khi doanh nghiệp sử dụng HĐĐT điều quan trọng đầu tiên phải đảm bảo là sản phẩm, công nghệ đó được thiết kế đơn giản, thân thiện với người dùng, có hướng dẫn sử dụng dịch vụ kèm theo; không cần phải sử dụng những phần mềm đặc biệt và phải phù hợp với đặc thù của doanh nghiệp.

Hạ tầng công nghệ công nghệ thông tin phải đáp ứng được tốc độ xử lý giao dịch, khả năng truy cập được mọi lúc, mọi nơi. Tiêu chuẩn của các giao dịch HĐĐT cũng như phần mềm hóa đơn đảm bảo yêu cầu liên thông tích hợp, duy nhất, bảo mật và có khả năng phát triển.

\section{Chuẩn chủ quan}

Chuẩn chủ quan đóng vai trò khá quan trọng đến việc doanh nghiệp có sử dụng HĐĐT hay không vì vậy phải xây dựng ý thức trách nhiệm khẳng định tầm quan trọng của hóa đơn điện tử trong bối cảnh hiện nay. Chính vì thế, khi tiến hành áp dụng sử dụng loại hóa đơn này, Nhà nước đã có một lộ trình rõ ràng đến 1/11/2020, để các doanh nghiệp có thể chuẩn bị một cách đầy đủ và chủ động hơn. Cần tập huấn, trang bị cho DN kiến thức về công nghệ thông tin và thường xuyên kiểm tra tình hình phát hành hóa đơn để phòng tránh các rủi ro có thể xảy ra

Sự tin tưởng và sự tương hỗ

Tăng cường phối kết hợp giữa cơ quan quản lý nhà nước, Nhà cung cấp phần mềm, Doanh nghiệp và khách hàng để tạo sự tin tưởng đối với hóa đơn điện tử

Chi Cục thuế Tân Phú cẩn đẩy mạnh tuyên truyền về hành lang pháp lý và lợi ích của việc phát hành HĐĐT, để các Doanh nghiệp hiểu rõ được những lợi ích của việc sử dụng hóa đơn điện tử và triển khai thực hiện sớm loại hình dịch vụ này. Song song đó là sự hỗ trợ tạo điều kiện của cơ quan quản lý nhà nước, không gây khó khăn cho Doanh nghiệp.

Nhà phát hành phần mềm cần có sự hỗ trợ chu đáo cho doanh nghiệp khi có các sự cố về giao dịch hay các vấn đề về vướng mắc sử dụng hoặc giao dịch trên nền tảng công nghệ mới của hóa đơn điện tử cần có sự hướng dẫn khắc phục nhanh chóng và kịp thời cho khách hàng.

Cơ quan thuế phải phân công cán bộ chuyên sâu hướng dẫn về văn hóa điện tử, thành lập website hỗ trợ khi doanh nghiệp muốn chọn HĐĐT mà chưa biết bắt đầu như thế nào, thiết lập đường dây nóng hỗ trợ HĐĐT.

Để đảm bảo hóa đơn điện tử được an toàn và bảo mật cần xây dựng hệ thống tự động xác minh chữ ký điện tử và gửi thông báo, mã xác nhận khi doanh nghiệp xuất hóa đơn.

\section{TÀI LIỆU THAM KHẢO}

1. Ajzen. (1991). The Theory of Planned Behavior University of Massachusetts at Amherst Cronin. University of Massachusetts at Amhers.

2. A. I. The Theory of Planned Behaviour. (1991). Organization Behaviour and Human Decision Processes. University of Massachusetts Amherst, 179-211.

3. Bruno Koch. (2019). The e-invoicing journey 2019-2025. Retrieved from: https://compacer.com/wpcontent/uploads/2019/05/Billentis_Report_compacer_single-sponsor.pdf

4. Davis F.D. (1989). Perceived usefulness, perceived ease of use, and user acceptance of information technologys. MIS Quarterly, Vol. 13 No. 3, pp. 319-340

5. Hoàng Trọng \& Chu Nguyễn Mộng Ngọc. (2008). Phân tích dữ liệu nghiên cứu với SPSS. NXB Hồng Đức. 
6. Lê Thị Kim Tuyết. (2011). Nghiên cứu động cơ sử dụng dịch vụ Internet Banking của người tiêu dùng tại thành phố Đà Nẵng. Tạp chí tài chính

7. Ngô Thị Bảo Châu. (2013). Phân tích các nhân tố ảnh hưởng đến hành vi mua sắm trực tuyến của người tiêu dùng thành phố Cần Thơ. Tạp chí khoa học truờng Đại Học Cần Tho.

8. Nguyễn Thị Hồng Liêm. (2016). Các yếu tố ảnh hưởng đến xu hướng chọn hóa đơn điện tử của doanh nghiệp. Tạp chí Tài chính, (6), 22-26

9. Nguyễn Thị Kim Anh. (2012). Nghiên cứu này là xác định các yếu tố ảnh hưởng đến thái độ đối với sử dụng ngân hàng trực tuyến của khách hàng tại thị trường TP. Tạp chí ngân hàng.

Ngày nhận bài: 25/02/2020

Ngày chấp nhận đăng: 27/03/2020 